\title{
O IBBD e a informação científica no Brasil*
}

\author{
The IBBD and scientific information in Brazil
}

\author{
Lydia de Queiroz Sambaquy \\ Mestra em Educação \\ Columbia University, Nova York, EUA \\ Instituto Brasileiro de Bibliografia e Documentação \\ (in memoriam)
}

\section{Resumo}

Criação do Instituto Brasileiro de Bibliografia e Documentação (IBBD). Significação do IBBD para a informação e documentação em ciência e tecnologia.

Palavras-chave

Documentação científica. Instituto Brasileiro de Bibliografia e Documentação - Brasil.

\section{Abstract}

Creation of the Brazilian Institute of Bibliography and Documentation (IBBD). IBBD's significance for information and documentation in science and technology.

\section{Keywords}

Scientific documentation. Brazilian Institute of Bibliography and Documentation - Brazil.

\section{INTRODUÇÃO}

É incontestável a importância dos registros culturais e de sua divulgação para o desenvolvimento dos processos civilizatórios, que modificam, paulatina e irreversivelmente, o comportamento e as realizações científicas, técnicas, educacionais e culturais de todos os povos.

As bibliotecas, como órgãos colecionadores e irradiadores dos conhecimentos de todos os tipos, adquiridos através do tempo e do espaço, apareceram há mais de 25 séculos, sendo suplementadas, mais tarde, pelos arquivos, museus e centros de informação especializados, que, atualmente, se contam aos milhares em seu conjunto.

A partir dos meados do século passado, com o desenvolvimento das bibliotecas universitárias e nacionais e dos centros e bibliotecas especializados em todos os campos do saber, bem como devido ao crescimento exponencial da produção literária e da documentação cientifica e tecnológica, surgiu a necessidade imperiosa da normalização dos procedimentos e métodos de trabalho nos órgãos de documentação, a fim de que pudessem trabalhar em conjunto, reunindo esforços, recursos financeiros e técnicas adequadas para

*Artigo orginalmente publicado no periódico Fórum Educacional, Rio de Janeiro, v. 12, n. 2, p. 31-41, abr./jun. 1988. Disponível em: http://bibliotecadigital.fgv.br/ojs/index.php/fe/article/view/61002/59219. 
conseguirem atingir o ideal comum, qual seja a realização do controle bibliográfico nas cidades, nos estados, nos países e em todo o mundo.

Isso quer dizer que as bibliotecas e centros de documentação precisam, atualmente, ter capacidade de conhecer o que já foi publicado ou documentado sob qualquer forma, para que possam ser obtidos, rápida e eficazmente, no momento exato em que forem necessários, informações e dados para estudos, trabalhos e pesquisas (BILLINGTON, s.d.).

É um desafio difícil e altamente representativo da capacidade de um Estado plenamente desenvolvido, ou em vias de desenvolvimento, conseguir dominar a produção intelectual mundial, para colocá-la à disposição de todos os que precisam aperfeiçoar seus conhecimentos,- resolver problemas técnicos, administrativos e científicos, conhecer o mundo através das inspirações filosóficas, artísticas e literárias, ou por meio de estudos históricos e sociais.

Graças aos planos elaborados pela Federação Internacional de Informação e Documentação (FID), pela Federação Internacional de Associações de Bibliotecas e Instituições (FIAB), pela Organização Interacional de Normalização (ISO) e, finalmente, mas não menos importante, pela Unesco (Organização das Nações Unidas para a Educação, Ciência e Cultura), vêm surgindo importantes iniciativas, em favor de um levantamento internacional da documentação disponível e em prol da acessibilidade dos dados $e$ informações nela contidos.

Assim, crescem paulatinamente projetos e esforços para o desenvolvimento de programas de cooperação e integração dos trabalhos de bibliotecas e centros de documentação em termos mundiais (ANDERSON, 1974).

O pioneirismo das bibliotecas de língua inglesa, nos Estados Unidos e na Inglaterra (que precedeu com as 91 regras de Pannizzi), seguido, de imediato, pela Alemanha e por muitos outros países europeus, merece toda uma história especial, pois foi de real importância seu esforço, no sentido da codificação de regras catalográficas e de classificação das coleções por sistemas numéricos elaborados para esse fim (BARBOSA,1976).

$O$ resultado desse entusiasmo pelo estabelecimento de sistemas e/ou redes de bibliotecas trabalhando em comum acordo, de forma cooperativa, teve pioneiros notáveis e efeitos espetaculares, que podem ser apreciados hoje em dia em todo o mundo, através de centenas de bases e bancos de dados a serviço da informação e documentação científica da educação e da cultura (SCHMITTROTH JR. et alii, 1983).

O Brasil despertou muito cedo para esse ideal, pelo menos teoricamente, como pode ser visto no Projeto de Regulamento para a Biblioteca Nacional, 1911-22, do então Diretor Manuel Cícero Peregrino, onde foi planejada estreita cooperação com o Instituto Internacional de Bibliografia, de Bruxelas, hoje FID, com sede em Haia (SILVA, s.d.; SAMBAQUY, 1956).

\section{CRIAÇÃO DO IBBD}

$\mathrm{Na}$ década de 50, havia, no Brasil e no mundo, o maior interesse pelo aperfeiçoamento da tecnologia da informação em todas as suas formas. A indústria do pósguerra, na Inglaterra e em vários países, tinha grande empenho na obtenção de know-how que promovesse o progresso econômico e, nessa época, já era bem compreendida a importância das bibliotecas como fundos de informações e dados imprescindíveis ao desenvolvimento de pesquisas e projetos e à produção de bens em geral. 
Nessa oportunidade, existia no Brasil grande entusiasmo por projetos que contribuíssem para o desenvolvimento dos trabalhos científicos e tecnológicos. Cesare Mansueto Giulio Lattes havia regressado ao país em 1949, vitorioso e honrado por sua importante participação, com Eugene Gardner, na descoberta do méson pesado e estava em vias de ser constituído, praticamente em sua homenagem, o Centro Brasileiro de Pesquisas Físicas, CBPF, no Rio de Janeiro. Ao mesmo tempo, o Alm. Álvaro Alberto da Motta e Silva, com a assistência do Departamento Administrativo do Serviço Público e da Academia de Ciência, estabelecia o Conselho Nacional de Pesquisas, atual Centro Nacional de Desenvolvimento Científico e Tecnológico, CNPq, e, nele, como órgão de eminente importância, o Instituto Brasileiro de Bibliografia e Documentação, IBBD (Simpósio sobre Biblioteconomia e Documentação Científica,1950; SAWAYA, s.d.; SAMBAQUY, 1955 e 1957). ${ }^{1}$

Luiz Simões Lopes, na direção da Fundação Getúlio Vargas, de há muito vinha batalhando no sentido de estabelecer uma rede de informações bibliográficas no país, tendo por base a formação de catálogos coletivos nacionais, aquisições planificadas e sistemas cooperativos de catalogação, desenvolvendo programas como os hoje denominados Controle Bibliográfico Universal (CBU) e Acessibilidade Universal de Publicações (UAP) disponíveis no país e no mundo, com resultados altamente compensadores (SAMBAQUY, [1980]).

Foi justamente a ele que Cesar Lattes sugeriu a ida ao exterior de técnicos que estudassem, em vários países da Europa e da América do Norte, o funcionamento dos centros de documentação e informação já, então, em plena atividade.

Junto com a Profa. Jannice Monte-Mór tivemos o privilégio de ser beneficiadas com extenso e proveitoso programa de estudos na Europa e América do Norte, como fellows da Unesco, quando obtivemos conhecimentos valiosos quanto à política, já então adotada, de procurar o domínio integral dos trabalhos intelectuais produzidos em todo o mundo e o estabelecimento da capacidade da obtenção de dados e informações em tempo ótimo e, ainda, quanto à aquisição de orientação prática, na constatação da realização de experiências, pesquisas e trabalhos, onde quer que estivessem estabelecidos ou em funcionamento centros de informação científica e tecnológica bem-sucedidos. Essa viagem foi realizada sob a orientação da Unesco (Paris), do British Council (Londres) e do International Institute of Education (Nova lorque).

É justo lembrar a atuação do nosso embaixador na Unesco na época, o Dr. Paulo Estevão Berredo Carneiro, para que fosse efetivado o plano que ele mesmo havia proposto pela primeira vez em São Paulo, durante o magnífico discurso de encerramento da Conferência pelo Desenvolvimento de Bibliotecas Públicas na América Latina, realizada em outubro de 1951 (UNESCO, 1952).

Nessa oportunidade, propôs que fosse escolhida, no Brasil, como órgão a ser assistido pelos programas para a América Latina da Unesco, a Biblioteca Pública do Paraná, cuja construção do novo prédio estava planejada pelo Governador Bento Munhoz da Rocha. A biblioteca pública-modelo que teria o suporte da Unesco, contudo, foi destinada à cidade de Medellín, na Colômbia. Abordou também a possibilidade de escolher o Brasil para sede do Centro de Documentação Científica e Tecnológica, mas, nessa época, o México já havia sido escolhido (ENCYCLOPEDIA OF LIBRARY AND INFORMATION SCIENCE, 1976).

Mesmo assim, o IBBD foi planejado e estabelecido pelo CNPq, com a colaboração valiosa da Fundação Getúlio Vargas e da Unesco. Sua constituição foi estudada por uma comissão designada pelo CNPq e integrada por: Joaquim da Costa Ribeiro, Octacílio Pinto

\footnotetext{
${ }^{1}$ O CNPq foi criado pela Lei no 1.310 . de 15 de Janeiro de 1951.
} 
Cordeiro de Souza e Mario Vianna Dias, representando o CNPq; Jorge Oscar de Mello Flores, Octacílio Silva Leal e Lydia de Queiroz Sambaquy, pela FGV.

A Exposição de Motivos no 426, de 5 de janeiro de 1954, assinada pelo Alm. Álvaro Alberto da Motta e Silva presidente do CNPq, e pelo Dr. Luiz Simões Lopes, presidente da FGV, foi submetida à apreciação do Presidente Getúlio Vargas, levando à criação do IBBD em 27 de fevereiro do mesmo ano e à sua instalação à Av. Gen. Justo, 171, 3ㅇ e 4음 and., no Rio de Janeiro (BRASIL, 1954).

A Profa. Jannice Monte-Mór escreveu pormenorizadamente sobre as iniciativas e trabalhos realizados para a concretização da idéia da criação do IBBD, em ensaio remetido ao IBICT (Instituto Brasileiro de Informação em Ciência e Tecnologia) em 7 de março de 1987 (MONTE-MÓR, 1986).

Cabia ao IBBD, fundamentalmente, segundo a letra do seu regimento, promover o intercâmbio de informações entre as instituições de pesquisas e divulgar, no Brasil e no estrangeiro, os trabalhos técnico-cient/1icos brasileiros.

\section{SIGNIFICAÇÃO DO IBBD PARA A INFORMAÇÃO E DOCUMENTAÇÃO EM CIÊNCIA E TECNOLOGIA}

O primeiro regimento do CNPq (BRASIL, 1966), aprovado pelo Decreto № 29.433, de 4 de abril de 1951, estabeleceu, em seu art. 74, que o "o Conselho Nacional de Pesquisas promoverá intercâmbio de informações bibliográficas pelos meios mais adequados, auxiliará o desenvolvimento das bibliotecas dos institutos de pesquisa e promoverá a formação de bibliotecas especializadas, onde julgar conveniente".

Para atender a esses objetivos do CNPq e tentar estabelecer uma eficiente rede de informações e documentação, a fim de bem servir ao desenvolvimento da pesquisa técnicocientífica em todo o país, foram realizados muitos estudos e reuniões sobre o objeto e as tarefas do IBBD.

Considerando o estado de abandono em que se encontrava a maioria das bibliotecas especializadas existentes no Brasil, desatualizadas, sem recursos para complementação de suas coleções e funcionando como departamentos estanques e sem uniformização em seus processos de catalogação, é importante destacar que o IBBD, desde o início, precisou organizar-se, tendo por base três tipos de serviços: serviços-fins, programados para atender diretamente aos cientistas e estudiosos, bem como às bibliotecas, principalmente as acadêmicas e científica; serviços de infra-estrutura técnica e, naturalmente, serviços-meios de administração em geral.

O seu objetivo maior deveria ser alcançado pelo Serviço de Informações TécnicoCientíficas, destinado a atender às necessidades de informações e dados apresentados diretamente pelos pesquisadores e estudiosos ou através de suas respectivas bibliotecas e centros de informação. Este serviço foi subdividido em três setores:

1. Seção de Informações e Intercâmbio, destinado, especialmente, aos cientistas e técnicos, pelo qual poderiam resolver seus problemas de localização e obtenção de obras desejadas;

2. Seção de Pesquisas Bibliográficas, que tinha por incumbência prestar o melhor serviço possível de busca de informações documentadas, bem como de compilação de bibliografias solicitadas por cientistas, tecnologistas e estudiosos em geral, enviando-lhes cópias dos textos de que necessitassem e cometendo-lhes, a pedido, dados bibliográficos, bem corno traduções de trabalhos científicos e tecnológicos em qualquer idioma; 
3. Seção de Assistência Técnica, incumbida de promover o treinamento de bibliotecários, assim corno de estudantes e diplomados em nível superior, desejosos de se aperfeiçoarem na técnica da pesquisa bibliográfica e documental em seus campos de especialização. Esse treinamento compreendia principalmente cursos de pesquisas bibliográficas em ciências médicas e biologia, química, física, matemática, etc., que, mais tarde, obtiveram o reconhecimento da Universidade do Brasil e hoje, por convênio com o IBICT, integram a Universidade Federal do Rio de Janeiro (UFRJ).

O IBBD promoveu também palestras, conferências, seminários, alguns com o patrocínio da Sociedade Brasileira para o Progresso da Ciência (SBPC), tendo participado de muitas reuniões de estudo promovidas por instituições internacionais.

Era idéia básica do Instituto aproveitar seu potencial de trabalho para contribuir para a melhoria dos serviços de informação, documentação e biblioteconomia especializada no Brasil. Cada biblioteca de maior importância passaria a funcionar como um elo de uma cadeia, em íntima colaboração com o IBBD e com as instituições nacionais e estrangeiras contatadas por ele, garantindo a maior perfeição nas buscas bibliográficas, a fim de que cada trabalho de pesquisa fosse secundado, de maneira fácil e exata, por informações que seriam obtidas num piscar de olhos.

Para isso, seria preciso renovar a mentalidade então vigente nas bibliotecas e centros de documentação, convencendo-se a todos de que sem cooperação integral, participação generosa e mudanças corajosas e definitivas de métodos e sistemas de trabalho, seria impossível a obtenção do desiderato maior do CNPq/IBBD.

Com esta finalidade foram criados vários serviços de infra-estrutura técnica, como: o Serviço de Intercâmbio de Catalogação, realizado como o auxílio da Fundação Getúlio Vargas, que, em colaboração com o Departamento Administrativo do Serviço Público (DASP) e o Departamento de Imprensa Nacional (DIN), desde 1942, já incluía 200 bibliotecas participantes de todo o país; o Serviço de Catálogo Coletivo (de livros e periódicos), que reunia a coleção completa de todos os catálogos coletivos que foram obtidos de outros países, além do Catálogo coletivo nacional, que estava em execução, com a contribuição das bibliotecas mais representativas de todo o Brasil, entre as quais a própria Biblioteca Nacional, no que se referia a monografias por ela catalogadas.

O Catálogo coletivo de publicações periódicas existente nas bibliotecas brasileiras que chegou a ser automatizado a partir de 1968, com a colaboração do Centro Brasileiro de Pesquisas Físicas e do Instituto Nacional de Pesquisas Espaciais de São José dos Campos, continua a existir como uma preciosa obra de referência, mantido pelo IBICT e distribuído em microfichas muito utilizadas pelas bibliotecas de todo o país.

Este catálogo, que relaciona, principalmente, seriados científicos e técnicos, em 1965 já incluía 33.507 títulos, correspondentes a 110.000 coleções de 303 bibliotecas participantes. Existe, ainda, em muitas bibliotecas, impresso em seis volumes, correspondentes aos anos de 1970-79 (Catálogo coletivo do IBBD, 1955; IBBD, 1955, 197071, 1977 e 1985).

O Catálogo coletivo de livros, que, em 1965, incluía 1.500 .000 títulos existentes em 328 bibliotecas, e o depósito de fichas bibliográficas impressas pelo Serviço de Intercâmbio de Catalogação desapareceram, como por encanto, depois da transformação do IBBD em IBICT.

Graças ao trabalho desenvolvido pelo IBBD em relação ao Catálogo coletivo, o Brasil foi dividido em nove regiões, que organizaram seus próprios catálogos e colaboraram intensamente com o Instituto, através da Comissão de Catálogo Coletivo, em reuniões 
regulares e decisões em, comum acordo. Teve início, então, a primeira tentativa de projeto de aquisição planificada, tendo em vista a complementação e atualização das bibliotecas científicas e técnicas com maior economia e melhores resultados (IBBD, 1962). ${ }^{2}$

Outro programa de fundamental importância para a informação e documentação sobre a produção científica e tecnológica brasileira foi realizado pelo IBBD, através do seu Serviço de Bibliografia, agora extinto porque o IBICT, paulatinamente, foi deixando de publicar as Bibliografias brasileiras correntes técnico-científicas, como a Bibliografia da Amazônia e as Bibliografias brasileiras de agricultura (1956-68), botânica (1950-64), ciências sociais (continuação da Bibliografia econômico-social da Fundação Getúlio Vargas, publicada no período 1954-64), matemática e física (1900-60), química (1957-65) e zoologia (1950-63).

Assim como a da Amazônia, estavam sendo compiladas algumas bibliografias científicas correntes, de interesse especial para as pesquisas em processo, como a do curare, da medicina tropical, da bouba, da doença de Chagas, da febre amarela, da leishmaniose, da malária e da esquistossomose. Compiladas, a pedido, no período a que se refere o Relatório Anual do IBBD de 1966, há inúmeras bibliografias especiais, limitadas pelos seus usuários a determinado período e assunto geralmente muito específico (IBBD, 1965).

Na verdade, não é possível a avaliação efetiva do progresso realizado pela ciência e tecnologia em um país senão através do conhecimento de sua produção bibliográfica e documentos culturais.

O Laboratório de Reproduções Fotográficas (LRF) do IBBD atuava em regime de intenso trabalho, porque era grande o interesse de pesquisadores e estudiosos em obter cópias e/ou ampliações dos microfilmes produzidos pelo próprio Instituto ou recebidos de bibliotecas brasileiras e estrangeiras. Este laboratório chegou a possuir uma máquina de ampliação automática de microfilmes, denominada Filmorex, importada da França.

O Serviço de Publicações, cujo equipamento maior foi doado ao IBBD pela Universidade Federal da Bahia, serviu eficientemente ao Instituto e, diretamente, ao CNPq.

Filiado à Federação Internacional de Documentação, hoje denominada Federação Internacional para a Informação e Documentação, da qual passou a ser membro nacional, o Instituto promoveu, com sucesso, a 26ạ Conferência Geral da FID, na sede da Escola Naval do Rio de Janeiro, onde ficaram hospedados todos os participantes, vindos de 25 países. Nessa oportunidade, foi estabelecida a FID/CLA (Comissão Latino-Americana da FID), sendo o presidente do IBBD eleito presidente desta primeira Comissão Regional da Federação.

O IBBD funcionou sempre com especial vocação para realizar trabalhos tão automatizados quanto possível. Por ocasião da Conferência da FID, foi feita uma ligação por telex entre a biblioteca do IBBD e a Escola Naval, sem dúvida a primeira tentativa de ligação das bibliotecas com seus usuários por telecomunicação de que se tem conhecimento no Brasil.

Pouco tempo depois, o IBBD passou a fazer uso intensivo do Retemec (Rede de Telecomunicações do MEC), para comunicar-se com as principais universidades brasileiras onde funcionavam os Centros Regionais de Catálogos Coletivos.

Muito se poderia dizer sobre os trabalhos projetados e realizados pelo IBBD para o desenvolvimento de programas de atividades que contribuíssem direta e poderosamente para o controle e divulgação da informação científica no país e para facilitar os empreendimentos do CNPq em todos os campos do conhecimento.

\footnotetext{
2 O prefácio que Lydia de Queiroz Sambaquy fez para e edição preliminar de Bibliotecas especializadas brasileiras: guia para intercâmbio bibliográfico (IBBD, 1962) apresenta o Programa para aquisição Planificada.
} 
Vale, nesse sentido, mencionar a publicação Guia das Pesquisas e Levantamentos em Processo no Brasil, que procurava arrolar todos os trabalhos científicos e tecnológicos programados e em desenvolvimento no país (IBBD, 1964 e 1968-70).

A biblioteca do IBBD foi destinada principalmente a colecionar obras de referência sobre todos os campos da ciência e tecnologia, obras geralmente dispendiosas que não são obtidas, de maneira sistemática, pelas melhores e mais atualizadas bibliotecas do Brasil. Capacitou-se, assim, a colocar à disposição dos estudiosos e das outras bibliotecas e centros de documentação, bibliografias, guias, catálogos e periódicos, que podiam responder sobre o que havia de mais moderno no Brasil e no mundo e que serviam a todas as instituições brasileiras interessadas no progresso científico e tecnológico. Essas coleções foram dispersadas pelas bibliotecas especializadas selecionadas pelo IBICT, e hoje ninguém sabe onde se encontram, estando muitas delas imprestáveis por sua desatualização e inacessibilidade. Mesmo nas melhores bibliotecas do país, as coleções de referência para assuntos científicos estão com a defasagem de, pelo menos, três anos, o que em ciência atualmente representa muito tempo.

Para terminar, vale fazer lembrar que o nome do IBBD ficou muito estimado no país e no exterior, principalmente na América Latina, que enviou ao Brasil bibliotecários e documentalistas para cursos e estágios especiais neste Instituto. Muitas bibliotecas e centros de documentação receberam assistência técnica direta do IBBD.

Concluímos com o registro do depoimento do grande patrono do IBBD, o Alm. Álvaro Alberto da Motta e Silva, que, em sua obra À margem da ciência (MOTTA E SILVA, 1960-70), comenta as dificuldades encontradas na localização da documentação necessária ao seu valioso trabalho, dizendo: "Não faltou, contudo, o elevado espírito de cooperação de parte do Instituto Brasileiro de Bibliografia e Documentação, que, com a usual eficiência, empreendeu longos e pacientes esforços para a indispensável obtenção de obras, fotocópias e microfilmes, em fontes nacionais e estrangeiras."

\section{REFERÊNCIAS}

ANDERSON, Dorothy. Universal bibliographic control. Paris, Unesco, 1974, 65p. (Intergovemmental Conference on the Planning of Natioj1al Documentation, Library and Archives Infrastructures, Paris, 23-27 Sep., 1974.)

BARBOSA, Alice Príncipe. Novos rumos da catalogação. Org., rev. e atualiz. Elza Lima e Silva Maia. Rio de Janeiro, BNG/Brasilart, 1976, 246p.

BILLINGTON, James $\mathrm{H}$. Tuming information into knowledge and distilling knowledge into wisdom. Excerpts of remarks by librarian of Congress at a press conference on Sept. 15, 1987. Library of Congress Information Bulletin, Washington, D.C., v. 46, n. 43, p. 459-463, s.d.

BRASIL. Leis, decretos, etc. Instituto Brasileiro de Bibliografia e Documentação. Decreto no 35.124, de 27 de fevereiro de 1954; Decreto no 35.430, de 29 de abril de 1954. Rio de Janeiro, Conselho Nacional de Pesquisas, 1954, 12p. 
BRASIL. Lei no 4.533, de 8 de dezembro de 1964 (D.O. de 15/12/64); Decreto no 56.122, de 27 de abril de 1965 (0.0. de 5/7/65); Regimento cio CNPq (D.O.) de 1/2/66). Rio de Janeiro, IBBD, 1966. 52p.

CATÁLOGO COLETIVO DO IBBD. IBBD Boletim Informativo. Rio de Janeiro, v.1, n. 2, p. 52, mar./abr. 1955.

ENCYCLOPEDIA of library and information science. New York; Basel, M. Kekker, 1976. v. 18, p. 13.

IBBD. Bibliotecas especializadas brasileiras: guia para intercâmbio bibliográfico. Rio de Janeiro, 1962, 376p.

IBBD. Catálogo coletivo de publicações periódicas de ciência e tecnologia. Rio de Janeiro, Serviço de Publicações, 1970-71. 2v. (Fontes de informação, 4.)

IBBD. Catálogo coletivo de publicações periódicas em ciências agrícolas e naturais. Rio de Janeiro, 1955. 2v. (Fontes de Informação, 12.)

IBBD. Catálogo coletivo de publicações periódicas em ciências biomédicas. Rio de Janeiro, 1977, 2v.

IBBD. Guia das pesquisas e levantamentos em processo no Brasil. Rio de Janeiro, 1964, $425 p$.

IBBD. Pesquisas em processo no Brasil, 1967-1969. Rio de Janeiro, Serviço de Publicações, 1968-70. 3v. (Fontes de informação, 1).

IBBD. Plano quinquenal de 1966-1970, elaborado por Lydia de Queiroz Sambaquy. Rio de Janeiro, CNPq/IBBD, 1965. p. 11.

IBICT. 30 anos de informação em C\& T no Brasil. Catálogo de publicações. Brasília, 1985. $31 p$.

MONTE-MÓR, Jannice de Mello. Memória para a história da criação do Instituto Brasileiro de Bibliografia e Documentação (IBBD). Rio de Janeiro, FGV, 1986,6 f. datilogr.

MOTTA E SILVA, Álvaro Alberto da. À margem da ciência. Rio de Janeiro: Imprensa Nacional, 1960-70. v. 1, p. XXIII-XXIV.

SAMBAQUY, Lydia de Queiroz. Luiz Simões Lopes e as realizações brasileiras no campo de biblioteconomia e documentação. Rio de Janeiro, FGV, [1980] xerox.

SAMBAQUY, Lydia de Queiroz. Manuel Cícero Peregrino da Silva. IBBD Boletim Informativo, Rio de Janeiro, v. 2, n. 5, p. 234-252, set./out. 1956. 
SAMBAQUY, Lydia de Queiroz. O Instituto Brasileiro de Bibliografia e Documentação e a informação científica no Brasil. BBD Boletim Informativo, Rio de Janeiro, v. 3, n. 5/6, p. 25360, set./dez. 1957.

SAMBAQUY, Lydia de Queiroz. Os problemas bibliográficos do cientista e o IBBD. IBBD Boletim Informativo, Rio de Janeiro, v. 1, n. 2, p. 33-34, mar./abr.1955.

SAWAYA, Paulo. Contribuição da biblioteconomia para o progresso científico. IBBD Boletim Informativo. Rio de Janeiro, v. 3, n. 3/4, p. 143-153. maio/ago.1959.

SCHMITTROTH JR. John et alii (Ed.). Encyclopedia of information systems and services. 5 . ed. Detroit, Gale Research, 1983.

SILVA, Luiz Antônio Gonçalves da. A institucionalização das atividades de informação científica e tecnológica no Brasil: o caso do Instituto Brasileiro de Bibliografia e Documentação (IBBD). Brasília, Faculdade de Estudos Sociais Aplicados, Universidade de Brasília, 1987. Tese de mestrado em Biblioteconomia e Documentação. Universidade de Brasília, Brasília. $226 \mathrm{f}$. datilogr. (Esta dissertação foi recebida quando Fórum Educacional estava em sua fase final de produção, tendo sido acrescentada, como obra que merece ser conhecida, por cortesia especial da direção desta revista.)

SILVA, Manuel Cícero Peregrino da. Projecto de regulamento para a Bibliotheca Nacional. [Rio de Janeiro], s. ed., s.d. (BN Mss. 1.33.25.1. Decreto no 8.835, de 11 de julho de 1911.).

SIMPÓSIO SOBRE BIBLIOTECONOMIA E DOCUMENTAÇÃO CIENTÍFICA. São Paulo, Conselho Nacional de Pesquisas; Rio de Janeiro, Instituto Brasileiro de Bibliografia e Documentação Científica (SIC); São Paulo, Associação Paulista de Bibliotecários, 1950. Anais [...] São Paulo, SBPC, 1950. 106 p. mimeogr.

UNESCO. Development of public libraries in Latin America: the São Paulo Conference. Paris, Unesco, 1952, 192 p. il.

\section{AGRADECIMENTOS}

Agradecemos ao periódico Fórum Educacional da Fundação Getúlio Vargas (FGV), ISSN 01009591, pela concessão à Ciência da Informação em Revista do direito de republicar e divulgar este artigo, nos termos da Licença Creative Commons 4.0. 